\title{
A Nonlinear Sliding Mode Controller for IPMSM Drives with an Adaptive Gain Tuning Rule
}

\author{
Jin-Woo Jung*, Dong Quang Dang*, Nga Thi-Thuy Vu*, Jackson John Justo*, Ton Duc Do*, Han Ho Choi*, \\ and Tae Heoung $\mathrm{Kim}^{\dagger}$ \\ *Division of Electronics and Electrical Engineering, Dongguk University, Seoul, Korea \\ ${ }^{\dagger}$ Dept. of Electrical Engineering, Engineering Research Institute, Gyeongsang National University, Jinju, Korea
}

\begin{abstract}
This paper presents a nonlinear sliding mode control (SMC) scheme with a variable damping ratio for interior permanent magnet synchronous motors (IPMSMs). First, a nonlinear sliding surface whose parameters change continuously with time is designed. Actually, the proposed SMC has the ability to reduce the settling time without an overshoot by giving a low damping ratio at the initial time and a high damping ratio as the output reaches the desired setpoint. At the same time, it enables a fast convergence in finite time and eliminates the singularity problem with the upper bound of an uncertain term, which cannot be measured in practice, by using a simple adaptation law. To improve the efficiency of a system in the constant torque region, the control system incorporates the maximum torque per ampere (MTPA) algorithm. The stability of the nonlinear sliding surface is guaranteed by Lyapunov stability theory. Moreover, a simple sliding mode observer is used to estimate the load torque and system uncertainties. The effectiveness of the proposed nonlinear SMC scheme is verified using comparative experimental results of the linear SMC scheme when the speed reference and load torque change under system uncertainties. From these experimental results, the proposed nonlinear SMC method reveals a faster transient response, smaller steady-state speed error, and less sensitivity to system uncertainties than the linear SMC method.
\end{abstract}

Key words: Interior Permanent Magnet Synchronous Motor (IPMSM), Nonlinear Sliding Surface, Sliding Mode Controller (SMC), Sliding Mode Observer (SMO), Speed Control, System Uncertainties

\section{INTRODUCTION}

With the well-known advantages of a compact structure, small size, high efficiency, low noise, and robustness, permanent magnet synchronous motors (PMSMs) have become more popular in speed and motion control applications. Structurally, PMSMs can be divided into two major categories: i) surface-mounted PMSMs (SPMSMs) and ii) interior PMSMs (IPMSMs). Due to their geometrical differences, IPMSMs have the following advantageous characteristics when compared to SPMSMs: higher speed operation and higher torque production [1], [2]. The former advantage results from a more robust rotor structure with magnets buried in the rotor core, while the latter one uses the

Manuscript received Jul. 16, 2014; accepted Feb. 13, 2015

Recommended for publication by Associate Editor Shihua Li.

${ }^{\dagger}$ Corresponding Author: ktheoung@gnu.ac.kr

Tel: +82-55-772-1717, Gyeongsang Nat'l University

*Division of Electronics and Electrical Engineering, Dongguk University, Korea reluctance torque as well as the electromagnetic torque.

Because of these properties, IPMSMs are extensively applied for high-performance variable-speed motor drives such as industrial robots, computer-controlled machine tools, household goods, electric vehicles (EVs), and plug-in hybrid electric vehicles (PHEVs) [3]. Despite their inherent advantages, the accurate speed control of IPMSM drives presents some difficult challenges in the presence of nonlinear coupling terms [4]. In addition, system uncertainties such as external disturbances and motor parameter variations can considerably deteriorate the control performances [5]. Accordingly, it is not easy for conventional PI controllers or LQ regulators to achieve good performance for IPMSM drives under the system uncertainties stated above [6].

In recent years, some advanced control methods such as adaptive control [7], fuzzy control [8] and [9], and neural network control [10] have been developed to satisfy the strict control requirements of IPMSM drive systems. In [7], an 
adaptive self-tuning MTPA vector control is presented to enhance the performance of IPMSM drives. This method is simple and robust to variations of system parameters. However, it is only introduced to control the currents. More recently, in [8], an online loss-minimization algorithm based on a fuzzy logic controller is developed for IPMSM drives to yield a high efficiency and a high dynamic performance over a wide speed range. In addition, in [9], the authors developed a simple fuzzy logic control strategy which is utilized as a speed controller with a reduced computational burden to achieve high performance for an IPMSM above its rated speed. However, these schemes [8] and [9] do not address uncertainty problems. A neuro-network control method is introduced in [10] to precisely control the speed of IPMSM driving systems. In this scheme, the system control achieves good performance and the system uncertainty problem is well solved. However, this approach requires burdensome computation due to a complex online algorithm.

Among the advanced control methods, the sliding mode control (SMC) is superior in comparison with the others because of its robustness. The SMC is less sensitive to parametric uncertainties, unmodeled dynamics, and external disturbances. As a result, it can be invariant to uncertainties in many cases and it is able to handle the nonlinearity of manipulated plants. On the other hand, the challenging problems of the SMC are its chattering phenomenon, singularity, and sensitivity to mismatched uncertainties resulting from a discontinuous switching gain [11]. This has led to the suggestion of various techniques in the literature, particularly in the field of motor drives. In [12], a neural model based SMC is presented for the trajectory tracking control of dc motors. This controller ensures the stability and robustness of closed-loop systems in the absence of a plant model and in the presence of external perturbations. In [13], a high performance discrete-time sliding-mode control is presented for induction motor drives. This control structure includes an active disturbance estimator, in which a passive filter is replaced by another discrete-time sliding mode controlled subsystem in order to improve the system robustness and accuracy. However, the stability of the system is not mathematically proven. In the sliding mode control of PMSMs, both an integral sliding surface [14] and a differential sliding surface [15] are used to improve the system performance. By introducing a boundary layer strategy [16], the chattering phenomenon can be reduced at the expense of the robustness against parameter uncertainties. Therefore, it is important to make a good compromise between the robustness and the chattering reduction. In [15], the SMC is combined with a fuzzy logic control to mitigate the chattering phenomena. However, problems such as settling time and overshoot have not been clearly addressed. Thus, the time-varying sliding surface technique [17] is a good solution to the above mentioned problems of the SMC.
This paper presents a method to design an observer-based nonlinear sliding mode control (SMC) scheme for IPMSMs with a variable damping ratio. By using a nonlinear sliding surface which is designed based on the variable damping concept, the proposed nonlinear SMC can remarkably reduce the settling time without any overshoot. In order to make the proposed SMC more feasible with a fast convergence in finite time, the upper bound of the uncertain term is adaptively estimated since it cannot be directly measured or calculated. In this paper, the maximum torque per ampere (MTPA) control is incorporated to maximize the torque generation in the constant torque region. The stability of the nonlinear sliding surface as well as the nonlinear SMC is mathematically proven using the Lyapunov stability analysis. Next, a simple sliding mode observer is applied to estimate both the load torque and the system uncertainties. The validity of the proposed nonlinear SMC is experimentally demonstrated using a prototype IPMSM drive system with a TMS320F28335 DSP. The experimental results show that the proposed nonlinear SMC attains better control performance with a faster transient response, a smaller steady-state speed error, and less sensitivity than the corresponding linear SMC when the desired speed and load torque change under system parameter uncertainties.

\section{STATE SPACE MODEL OF AN IPMSM WITH SYSTEM UNCERTAINTIES}

\section{A. Dynamic Model of an IPMSM}

Applying Kirchhoff's voltage law $(K V L)$ to the $d q$-axis equivalent circuits of a three-phase IPMSM yields the following voltage equations in the synchronously rotating $d-q$ reference frame:

$$
\begin{gathered}
V_{q s}=R_{s} i_{q s}+L_{q s} \dot{i}_{q s}+\omega L_{d s} i_{d s}+\omega \lambda_{m} \\
V_{d s}=R_{s} i_{d s}+L_{d s} \dot{i}_{d s}-\omega L_{q s} i_{q s}
\end{gathered}
$$

where $V_{d s}$ and $V_{q s}$ are the $d q$-axis voltages, $i_{d s}$ and $i_{q s}$ are the $d q$-axis currents, $R_{s}$ is the stator resistance, $L_{d s}$ and $L_{q s}$ are the $d q$-axis inductances, $\omega$ is the electrical rotor speed, and $\lambda_{m}$ is the magnetic flux.

In addition, the electromagnetic torque can be obtained from the following electrical and mechanical equations:

$$
\begin{gathered}
T_{e}=\frac{3}{2} \frac{p}{2}\left[\lambda_{m} i_{q s}+\left(L_{d s}-L_{q s}\right) i_{d s} i_{q s}\right] \\
T_{e}=T_{L}+B \frac{2}{p} \omega+J \frac{2}{p} \dot{\omega}
\end{gathered}
$$

where $T_{e}$ and $T_{L}$ are the electromagnetic and load torques, $p$ is the number of poles, $B$ is the viscous friction coefficient, and $J$ is the rotor inertia.

Substituting (3) into (4) yields the following speed dynamic equation:

$$
\dot{\omega}=\frac{3}{2} \frac{p^{2}}{4} \frac{\lambda_{m}}{J} i_{q s}-\frac{B}{J} \omega-\frac{p}{2 J} T_{L}+\frac{3}{2} \frac{p^{2}}{4} \frac{L_{d s}-L_{q s}}{J} i_{q s} i_{d s}(5)
$$


By using (1)-(5), the dynamic model of the IPMSM can be expressed as:

$$
\begin{aligned}
& \dot{\omega}=k_{1} i_{q s}-k_{2} \omega-k_{3} T_{L}+k_{11} i_{d s} i_{q s} \\
& \dot{i}_{q s}=-k_{4} i_{q s}-k_{5} \omega+k_{6} V_{q s}-k_{10} \omega i_{d s} \\
& \dot{i}_{d s}=-k_{7} i_{d s}+k_{8} V_{d s}+k_{9} \omega i_{q s}
\end{aligned}
$$

where the $k_{1}$ to $k_{11}$ are the coefficients defined in [5]. In addition, taking into consideration the system uncertainties such as motor parameter variations, external disturbances, etc., the system model (6) can be rewritten as follows:

$$
\begin{aligned}
& \dot{\omega}=k_{1} i_{q s}-k_{2} \omega+k_{11} i_{d s} i_{q s}-k_{3} d_{1} \\
& \dot{i}_{q s}=-k_{4} i_{q s}-k_{5} \omega+k_{6} V_{q s}-k_{10} \omega i_{d s}-k_{6} d_{2} \\
& \dot{i}_{d s}=-k_{7} i_{d s}+k_{8} V_{d s}+k_{9} \omega i_{q s}-k_{8} d_{3}
\end{aligned}
$$

where $d_{1}, d_{2}$, and $d_{3}$ are uncertain components [5] that represent motor parameter variations and external disturbances.

Remark 1: The uncertain components $d_{1}, d_{2}$, and $d_{3}$ are unknown. However, they are assumed to be bounded, i.e., there exist the constants $\gamma_{1}, \gamma_{2}$, and $\gamma_{3}$ which satisfy $\left|d_{1}\right| \leq \gamma_{1}$, $\left|d_{2}\right| \leq \gamma_{2}$, and $\left|d_{3}\right| \leq \gamma_{3}$. These assumptions are reasonable because variations of motor parameters cannot be infinite.

\section{B. State Space Model of an IPMSM}

The state space variables are defined as:

$$
z_{1}=\omega-\omega_{d}, \quad z_{2}=\dot{z}_{1}, \quad z_{3}=i_{d s}-i_{d s d}
$$

where $\omega_{d}$ and $i_{d s d}$ are the desired values of $\omega$ and $i_{d s}$, respectively. In this paper, only the following assumptions are used: $\omega, i_{q s}$, and $i_{d s}$ are measurable and an external load torque $\left(T_{L}\right)$ is unavailable.

In IPMSMs, the reluctance torque is available because of the existing saliency $\left(L_{q s}>L_{d s}\right)$. If the desired $d$-axis current $i_{d s d}$ is kept at zero, it is not possible to utilize the potential reluctance torque. Thus, in order to maximize the torque generation of IPMSMs in the constant torque region and increase the efficiency of IPMSM drives, the armature current should be controlled according to the maximum torque per ampere (MTPA) trajectory operation. In this technique, the $d$-axis current reference is given by the equation (9) as in [4].

$$
i_{d s d}=-\frac{\left(L_{q s}-L_{d s}\right)}{\lambda_{m}} i_{q s}^{2}
$$

By taking the derivative of (8) and using (7), the following results are obtained:

$$
\begin{aligned}
\dot{z}_{1}= & z_{2} \\
\dot{z}_{2}= & -k_{1} k_{5} z_{1}-k_{2} z_{2}+k_{1} k_{6} V_{q s}-k_{1} k_{5} \omega_{d}-k_{1} k_{10} i_{d s} \omega-k_{2} \dot{\omega}_{d} \\
& +k_{11}\left(\dot{i}_{d s} i_{q s}+\dot{i}_{q s} i_{d s}\right)-k_{1} k_{4} i_{q s}-k_{1} k_{6} d_{2}-k_{3} \dot{d}_{1}-\ddot{\omega}_{d} \\
\dot{z}_{3}= & -k_{7} z_{3}+k_{8} V_{d s}+k_{9} \omega i_{q s}-k_{8} d_{3}-k_{7} i_{d s d}-\dot{i}_{d s d}
\end{aligned}
$$

Therefore, it is possible to set the following equations:

$$
\begin{aligned}
g_{2}= & -k_{1} k_{5} \omega_{d}-k_{1} k_{10} i_{d s} \omega-k_{2} \dot{\omega}_{d}+k_{11}\left(\dot{i}_{d s} i_{q s}+\dot{i}_{q s} i_{d s}\right) \\
& -k_{1} k_{4} i_{q s}-k_{1} k_{6} d_{2}-k_{3} \dot{d}_{1}-\ddot{\omega}_{d} \\
g_{3}= & -k_{8} d_{3}+k_{9} \omega \dot{i}_{q s}-k_{7} i_{d s d}-\dot{i}_{d s d}
\end{aligned}
$$

Then the model (10) can be reduced to:

$$
\begin{aligned}
& \dot{z}_{1}=z_{2} \\
& \dot{z}_{2}=k_{1} k_{5} z_{1}-k_{2} z_{2}+k_{1} k_{6} V_{q s}+g_{2} \\
& \dot{z}_{3}=-k_{7} z_{3}+k_{8} V_{d s}+g_{3}
\end{aligned}
$$

From (12), the dynamic model of IPMSMs can be expressed in the state space form:

$$
\dot{z}=A z+B u+g
$$

where $z=\left[\begin{array}{lll}z_{1} & Z_{2} & z_{3}\end{array}\right]^{T}$

$$
A=\left[\begin{array}{ccc}
0 & 1 & 0 \\
k_{1} k_{5} & -k_{2} & 0 \\
0 & 0 & -k_{7}
\end{array}\right]=\left[\begin{array}{lll}
a_{11} & a_{12} & a_{13} \\
a_{21} & a_{22} & a_{23} \\
a_{31} & a_{32} & a_{33}
\end{array}\right],
$$

$$
B=\left[\begin{array}{ll}
0 & 0 \\
1 & 0 \\
0 & 1
\end{array}\right]=\left[\begin{array}{ll}
b_{11} & b_{12} \\
b_{21} & b_{22} \\
b_{31} & b_{32}
\end{array}\right], u=\left[\begin{array}{c}
u_{1} \\
u_{2}
\end{array}\right]=\left[\begin{array}{c}
k_{1} k_{6} V_{q s} \\
k_{8} V_{d s}
\end{array}\right], g=\left[\begin{array}{c}
0 \\
g_{2} \\
g_{3}
\end{array}\right]
$$

where $u$ is the control input. Now, the goal of this study is to design a control law $u$ for uncertain linear systems (13).

\section{Nonlinear SLIDING SURface DeSigN}

\section{A. Nonlinear Sliding Surface Design}

In this section, a nonlinear sliding surface is designed by using the principle of the variable damping concept. By changing the damping ratio of the system from its initial value to the final high value, the system response during the transient-state can be fast without overshoot while the chances of a singularity can be significantly minimized [11]. This idea can be described as follows.

The dynamic model (13) is rewritten in the following form:

$$
\begin{aligned}
& \dot{z}_{1}=z_{2} \\
& \dot{z}_{2}=a_{21} z_{1}+a_{22} z_{2}+u_{1}+g_{2} \\
& \dot{z}_{3}=a_{33} z_{3}+u_{2}+g_{3}
\end{aligned}
$$

The sliding surface is defined as:

$$
\begin{aligned}
\sigma & =\left[\begin{array}{l}
\sigma_{1} \\
\sigma_{2}
\end{array}\right]=S z=\left[\begin{array}{l}
S_{1} z \\
S_{2} z
\end{array}\right]=\left[\begin{array}{ccc}
F_{11}-\Phi(y) a_{12} P & 1 & 0 \\
F_{21}-\Phi(y) a_{13} P & 0 & 1
\end{array}\right] z \\
& =\left[\begin{array}{ccc}
F_{11}-\Phi(y) P & 1 & 0 \\
F_{21} & 0 & 1
\end{array}\right] z=\left[\begin{array}{c}
F_{11} z_{1}-\Phi(y) P z_{1}+z_{2} \\
F_{21} z_{1}+z_{3}
\end{array}\right]
\end{aligned}
$$

where $F=\left[\begin{array}{ll}F_{11} & F_{21}\end{array}\right]^{T}$ is a constant matrix which is designed based on the given settling time $t_{s}$ and damping ratio $\zeta_{1}$. In addition, $P$ is a positive constant that is chosen based on the desired final damping ratio $\zeta_{2}$ and it satisfies the following condition:

$$
\left(a_{11}-F^{T} A_{1}^{T}\right) P+P\left(a_{11}-A_{1} F\right)=-Q
$$




$$
F^{T} A_{1}^{T} P+P\left(A_{1} F\right)=Q
$$

where $Q$ is a positive weighting constant and $A_{1}=\left[\begin{array}{ll}a_{12} & a_{13}\end{array}\right]$ $=\left[\begin{array}{ll}1 & 0\end{array}\right]$.

It should be noted that (17) is an algebraic Lyapunov equation that is required to guarantee the stability of the proposed method. The function $\Phi(y)$ will be designed later and $y$ is the control output of the system.

$$
y=C z=\left[\begin{array}{lll}
1 & 0 & 0
\end{array}\right] z
$$

The selection of the function $\Phi(y)$ should satisfy the condition that as the output changes from the starting point to the origin, the value of the function $\Phi(y)$ decreases from 0 or a very small value to $-\beta$ where $\beta$ is a positive scalar. This is the necessary condition to make the sliding surface (15) nonsingular. In addition, the function $\Phi(y)$ should have the first degree differential equation in $y$.

In this study, the function $\Phi(y)$ is selected based on [16] with the following form:

$$
\Phi(y)=-\beta e^{-k y^{2}}
$$

where $k$ is a positive scalar that should be large enough to ensure a small initial value of $\Phi(y)$.

The stability of the sliding surface (15) is verified by the following theorem.

Theorem 1: Assume that there exists a matrix $F$ such that $\left(a_{11}\right.$ $-A_{1} F$ ) is stable and a positive definite matrix $P$ that is chosen based on the desired final damping ratio $\zeta_{2}$. Also, it satisfies the condition in (16) with the function $\Phi(y)$ defined by (19). Then the nonlinear sliding surface defined by (15) is stable.

Proof: Refer to the standard sliding mode control method presented in [18] and note that during the sliding mode, $\sigma=\dot{\sigma}=0$. From (14), it is easy to see that:

$$
\begin{aligned}
& z_{2}=-\left[F_{11}-\Phi(y) P\right] z_{1} \\
& z_{3}=-F_{21} z_{1}
\end{aligned}
$$

By using (20), the system dynamics (14) can be expressed by the following first-order equation:

$$
\dot{z}_{1}=-\left[F_{11}-\Phi(y) P\right] z_{1}
$$

For the dynamics (21), the Lyapunov function can be chosen as:

$$
V(z)=P z_{1}^{2}
$$

The derivative of $V(z)$ along with (21) is obtained as:

$$
\begin{aligned}
\dot{V}(z) & =2 P z_{1} \dot{z}_{1}=-2 P z_{1}\left[F_{11}-\Phi(y) P\right] z_{1} \\
& =2 z_{1}^{2}\left[-P F_{11}+\Phi(y) P^{2}\right]
\end{aligned}
$$

The condition represented by (17) implies that:

$$
\dot{V}(z)=z_{1}^{2}\left[-Q+2 \Phi(y) P^{2}\right]
$$

In this case, the properties of the function $\Phi(y)$ guarantee that it is a negative function. In addition, it can be concluded that the sliding mode dynamics (21) or the sliding surface (15) is asymptotically stable.

\section{B. Sliding Surface Parameters Design}

As indicated in (15), the sliding surface consists of linear and nonlinear parts. The function $\Phi(y)$ in the nonlinear part changes its value from zero to $-\beta$ when the output changes from the initial state to the origin. This reduction makes the damping ratio of the system increase from the initial value $\zeta_{1}$ to the final value $\zeta_{2}$. Consider the system represented by:

$$
\dot{z}_{1}=\left[a_{11}-A_{1} F+\Phi(y)\left(a_{12}^{2}+a_{13}^{2}\right) P\right] z_{1}=-\left[F_{11}-\Phi(y) P\right] z_{1}
$$

At the initial-state, by choosing a high value for the factor $k$, the function $\Phi(y)$ has a small value which is trivial. The damping ratio is determined by $F$. Thus, the matrix $F$ is designed for a low damping ratio $\zeta_{1}$. In this paper, the matrix $F$ is found by the pole placement approach with a given initial settling time and damping ratio.

At the steady-state, the output reaches the origin point and the function $\Phi(y)$ attains its final value $\Phi(y)=-\beta$. As a result, the nominal reduced-order dynamics (21) becomes:

$$
\begin{aligned}
\dot{z}_{1} & =\left[a_{11}-A_{1} F-\beta\left(a_{12}^{2}+a_{13}^{2}\right) P\right] z_{1} \\
& =\left[a_{11}-A_{1}\left(F+\beta A_{1}^{T} P\right)\right] z_{1}=\left(a_{11}-A_{1} M\right) z_{1}=-A_{1} M z_{1}
\end{aligned}
$$

where:

$$
M=F+\beta A_{1}^{T} P
$$

or

$$
A_{1}^{T} P-\frac{M-F}{\beta}=0
$$

The gain $M$ is calculated in a way that is similar to $F$ for the final damping ratio $\zeta_{2}$. It can be seen that in order to guarantee the stability of the closed-loop system, the matrix $P$ should satisfy the condition (16). In addition, to realize the desired damping ratio, the matrix $P$ needs to satisfy (28). However, it is difficult to find a matrix $P$ which satisfies both (16) and (28). Therefore, the condition in (28) may be moderated as follows:

$$
\left\|A_{1}^{T} P-\frac{M-F}{\beta}\right\| \leq \mu
$$

where $\mu>0$ is a sufficiently scalar. Using the Schur complement formula, the condition given by (29) can be rewritten in the form of a linear inequality as follows:

$$
\left[\begin{array}{cc}
\mu I & A_{1}^{T} P-\frac{M-F}{\beta} \\
\left(A_{1}^{T} P-\frac{M-F}{\beta}\right)^{T} & \mu I
\end{array}\right]>0
$$

By this approximation, the design goal is to find the matrix $P$ which minimizes $\mu$ such that:

$$
\begin{gathered}
P>0 \\
F^{T} A_{1}^{T} P+P A_{1} F>0
\end{gathered}
$$




$$
\left[\begin{array}{cc}
\mu I & A_{1}^{T} P-\frac{M-F}{\beta} \\
\left(A_{1}^{T} P-\frac{M-F}{\beta}\right)^{T} & \mu I
\end{array}\right]>0
$$

\section{NONLINEAR SLIDING Mode SPEED CONTROLLER DESIGN}

In the previous section, it was shown that there exists a nonlinear asymptotic stable sliding surface whose parameters change continuously with time. In this section, a nonlinear sliding mode speed control law is designed for systems (13) using the nonlinear sliding surface (15). The controller should guarantee the reaching condition to ensure that the system trajectory goes toward the sliding surface from any initial value and then slides along the sliding surface to the origin. Such a controller is introduced in the following theorem.

Theorem 2: Assuming that the uncertain term $g$ is bounded by a constant and there exists some positive constant $\gamma$ such that $\gamma \geq\|S\|\|g\|$. Consider the following nonlinear feedback control law:

$$
u=-(S A z+K \sigma+\gamma \operatorname{sign}(\sigma)+\dot{S} z)
$$

where $K$ is a positive scalar, and $\sigma$ is the nonlinear sliding surface given by (15). Then the state of the uncertain system (14) will converge to zero.

Proof: According to the standard sliding mode control [18], the sliding surface will approach zero in a finite amount of time if the sliding condition $\sigma^{T} \dot{\sigma}<0$ is guaranteed for all $\sigma(t) \neq 0$.

From (13), (15), and (34), the reaching condition can be derived as:

$$
\begin{aligned}
\sigma^{T} \dot{\sigma} & =\sigma^{T}(S \dot{S}+\dot{S} z)=\sigma^{T}[S(A z+B u+g)+\dot{S} z] \\
& =\sigma^{T}[S A z+S B u+S g+\dot{S} z]
\end{aligned}
$$

Since $S B=I$, where $I$ is an identity matrix, then (35) is rewritten as:

$$
\begin{aligned}
\sigma^{T} \dot{\sigma} & =\sigma^{T}[S A z+u+S g+\dot{S} z] \\
& =\sigma^{T}(S A z-S A z-K \sigma-\gamma \operatorname{sign}(\sigma)-\dot{S} z+S g+\dot{S} z) \\
& =\sigma^{T}(-K \sigma-\gamma \operatorname{sign}(\sigma)+S g) \\
& \leq-K\|\sigma\|^{2}-\gamma\left(\left|\sigma_{1}\right|+\left|\sigma_{2}\right|\right)+\left|\sigma_{1}\right| \cdot\left|S_{1} g\right|+\left|\sigma_{2}\right| \cdot\left|S_{2} g\right| \\
& \leq-K\|\sigma\|^{2}-\gamma\left(\left|\sigma_{1}\right|+\left|\sigma_{2}\right|\right)+\left|\sigma_{1}\right| \cdot\left\|S_{1}\right\| \cdot\|g\|+\left|\sigma_{2}\right| \cdot\left\|S_{2}\right\| \cdot\|g\| \\
& \leq-K\|\sigma\|^{2}-\gamma\left(\left|\sigma_{1}\right|+\left|\sigma_{2}\right|\right)+\left|\sigma_{1}\right| \cdot\|S\| \cdot\|g\|+\left|\sigma_{2}\right| \cdot\|S\| \cdot\|g\| \\
& \leq-K\|\sigma\|^{2}-\gamma\left(\left|\sigma_{1}\right|+\left|\sigma_{2}\right|\right)+\|S\| \cdot\|g\|\left(\left|\sigma_{1}\right|+\left|\sigma_{2}\right|\right) \leq-K\|\sigma\|^{2}
\end{aligned}
$$

According to (36), it is obvious that a stable sliding motion is induced in a finite amount of time. Then it completes the proof.

Remark 2: In (34), the control law $u$ contains the total derivative of the output. In this paper, this derivative can be easily calculated by using the robust differentiator given by Utkin [19]. In addition, the efficiency of these methods has been confirmed via many practical applications.

Remark 3: The value of $\gamma$ in the controller (34) is an unknown constant. This value can be obtained by the following simple adaptation law:

$$
\dot{\hat{\gamma}}=\frac{1}{\alpha} \sigma^{T} \operatorname{sign}(\sigma)
$$

where $\alpha$ is a positive scalar.

By using this adaptation law, the controller (34) becomes:

$$
u=-(S A z+K \sigma+\hat{\gamma} \operatorname{sign}(\sigma)+\dot{S} z)
$$

The validity of the control law (38) and the adaptation law (37) is confirmed by Lyapunov theory.

Define the adaptation error as:

$$
\tilde{\gamma}=\gamma_{d}-\hat{\gamma}
$$

where $\gamma_{d}$ is an unknown real value of $\gamma$, and $\gamma_{d} \geq\|S\|\|g\|$.

Choose Lyapunov function as:

$$
V_{1}=\frac{1}{2} \sigma^{T} \sigma+\frac{1}{2} \alpha \tilde{\gamma}^{2}
$$

The derivative of $V_{1}$ along with (39) is as follows:

$$
\dot{V}_{1}=\sigma^{T} \dot{\sigma}+\alpha \dot{\tilde{\gamma}} \tilde{\gamma}
$$

From (36), (39), and (40), the following is obtained:

$$
\begin{aligned}
\dot{V}_{1} & =\sigma^{T}(-K \sigma-\hat{\gamma} \operatorname{sign}(\sigma)+S g)-\alpha \dot{\hat{\gamma} \tilde{\gamma}} \\
& =\sigma^{T}\left(-K \sigma-\gamma_{d} \operatorname{sign}(\sigma)+S g\right)+\tilde{\gamma} \sigma^{T} \operatorname{sign}(\sigma)-\alpha \dot{\tilde{\gamma}} \tilde{\gamma} \\
& \leq-K\|\sigma\|^{2}+\tilde{\gamma}\left(\sigma^{T} \operatorname{sign}(\sigma)-\alpha \dot{\hat{\gamma}}\right)
\end{aligned}
$$

Hence, (37) guarantees that:

$$
\dot{V}_{1} \leq-K\|\sigma\|^{2} \leq 0
$$

\section{SYSTEM UnCERTAINTIES OBSERVER DESIGN}

It well known from (7) and (12) that the proposed nonlinear SMC contains load torque disturbances $T_{L}$ and system uncertainties $d_{1}$. These unknown disturbances can make the motor speed unstable during the transient-state. In addition, they can cause unexpected errors during the steady-state. Thus, in the speed tracking control of IPMSMs, the information on these disturbances is required. Thus, a simple sliding mode observer is used to estimate the load torque $T_{L}$ and system uncertainties $d_{1}$.

The first equation of (7) can be rewritten as:

$$
\dot{\omega}=k_{1} i_{q s}-k_{2} \omega+k_{11} i_{d s} i_{q s}-k_{3} d_{1}
$$

In order to design a sliding mode uncertainties observer, the following sliding surface is defined:

$$
\sigma_{o}=\omega-\hat{\omega}
$$

where $\hat{\omega}$ is an estimate of the rotor speed calculated from the following:

$$
\dot{\hat{\omega}}=k_{1} i_{q s}-k_{2} \omega+k_{11} i_{q s} i_{d s}+l \operatorname{sgn}(\omega-\hat{\omega})
$$

where $l>0$. 
The error dynamics of the sliding mode observer is given as:

$$
\dot{\sigma}_{o}=\dot{\omega}-\dot{\hat{\omega}}=-k_{3} d_{1}-l \operatorname{sgn}\left(\sigma_{o}\right)
$$

It is well known by the standard sliding mode method [26] that in the steady-state, $\sigma_{o}=\dot{\sigma}_{o}=0$. By using (40), it is easy to see that:

$$
k_{3} d_{1} \approx-\operatorname{lggn}\left(\sigma_{o}\right)
$$

The estimated uncertainties can be reconstructed as follows:

$$
\hat{d}_{1}=-\frac{1}{1+s \tau_{o}} \frac{l}{k_{3}} \operatorname{sgn}\left(\sigma_{o}\right)
$$

where $s$ is the Laplace variable, and $\tau_{o}$ is a sufficiently small filter time constant.

Remark 4: Actually, the discontinuous function sgn(y) may lead to an undesirable chattering phenomenon in the action of the sliding mode control (SMC). Thus, if $\operatorname{sgn}(y)$ is replaced by a continuous approximation such as $y /(\|y\|+\eta)$, where $\eta$ denotes a positive constant value, this chattering problem can be considerably attenuated. It should be noted that there is almost no performance difference between the approximated control law and the actual control law [15] as $\eta$ approaches zero. It should be noted that the chattering and mismatched uncertainties problems [20], can be mitigated by methods such as the homogeneity approach to high-order sliding mode control, the observer-based chattering suppression method, the state-dependent gain method, the equivalent control dependent gain method, the chattering frequency control method using a hysteresis loop, and the integral SMC presented in [21].

\section{NUMERICAL EXAMPLE}

In this section, a prototype IPMSM drive is used to verify the effectiveness of the proposed observer-based speed controller. The nominal parameters of the prototype IPMSM are specified as: rated power $P_{\text {rated }}=390 \mathrm{~W}$; rated torque $T_{\text {rated }}=1.5 \mathrm{~N} \cdot \mathrm{m}$; rated current $I_{\text {rated }}=4.3 \mathrm{~A}$; rated speed $\omega_{\text {rated }}$ $=2500 \mathrm{rpm} ; p=4 ; R_{s}=2.48 \Omega ; L_{q s}=113.91 \mathrm{mH} ; L_{d s}=$ $74.98 \mathrm{mH} ; \lambda_{m}=0.193 \mathrm{~V} \cdot \mathrm{s} / \mathrm{rad} ; J=0.00042 \mathrm{~kg} \cdot \mathrm{m}^{2}$; and $B=$ $0.0001 \mathrm{~N} \cdot \mathrm{m} \cdot \mathrm{s} / \mathrm{rad}$.

Using Remark 4 in Section V, the uncertainties observer represented by (42) becomes:

$$
\hat{d}_{1}=\frac{1}{1+s \tau_{o}} \frac{l}{k_{3}} \frac{\sigma_{o}}{\left|\sigma_{o}\right|+\eta_{o}}
$$

where $l=8000, \tau_{o}=0.01$, and $\eta_{o}=0.06$.

In order to calculate the sliding surface parameters, it is assumed that the initial settling time $t_{s 1}$ is $100 \mathrm{~ms}$ and that the initial damping ratio $\zeta_{1}$ is 1 . In addition, the matrix $F$ can be calculated using the pole placement technique which gives:

$$
F=\left[\begin{array}{c}
127.92 \\
0
\end{array}\right]
$$

Solving the Lyapunov function (16) with $Q=10^{-3}$, the resulting value of $P$ is obtained as $5.1 \times 10^{-6}$.

The nonlinear sliding surface is now built by using the above matrices $F$ and $P$ with $\beta=300$ and $k=50$ as:

$$
\sigma=S z=\left[\begin{array}{ccc}
F_{11}+300 e^{-50 y^{2}} P & 1 & 0 \\
F_{21} & 0 & 1
\end{array}\right] Z
$$

Again, by using the nonlinear sliding surface (52) and Remarks 3 and 4, the nonlinear sliding mode control law (34) can be expressed as:

$$
u=\left[\begin{array}{l}
u_{1} \\
u_{2}
\end{array}\right]=-\left(S A z+K \sigma+\hat{\gamma} \frac{\sigma}{\|\sigma\|+\eta}+\dot{S} z\right)
$$

where $K=250, \eta=0.1$, and $\alpha=1500$.

In addition, considering the estimated load torque and system uncertainties instead of their real values, the controller (53) can be rewritten as:

$$
\hat{u}=\left[\begin{array}{l}
\hat{u}_{1} \\
\hat{u}_{2}
\end{array}\right]=-\left(S A \hat{z}+K \hat{\sigma}+\hat{\gamma} \frac{\hat{\sigma}}{\|\hat{\sigma}\|+\eta}+\dot{S} \hat{z}\right)
$$

where:

$$
\begin{aligned}
& \hat{\sigma}=S \hat{z}=\left[\begin{array}{ccc}
F_{11}+300 e^{-50 y^{2}} P & 1 & 0 \\
F_{21} & 0 & 1
\end{array}\right] \hat{z}, \\
& \hat{z}=\left[\begin{array}{c}
z_{1} \\
\hat{z}_{2} \\
z_{3}
\end{array}\right]=\left[\begin{array}{c}
\omega-\omega_{d} \\
k_{1} i_{q s}-k_{2} \omega+k_{11} i_{d s} i_{q s}-k_{3} \hat{d}_{1} \\
i_{d s}-i_{d s d}
\end{array}\right]
\end{aligned}
$$

Finally, the control input signals to the space vector pulse-width modulation (SVPWM) are obtained as:

$$
V_{q s}=\frac{1}{k_{1} k_{6}} \hat{u}_{1}, \quad V_{d s}=\frac{1}{k_{8}} \hat{u}_{2}
$$

In order to verify the performance and effectiveness of the proposed observer-based nonlinear sliding mode controller, experiments are carried out with a prototype IPMSM drive system based on a TMS320F28335 DSP.

Fig. 1 shows an overall block diagram of the proposed observer-based nonlinear sliding mode speed control system. The hardware circuit consists of an IPMSM, a three-phase inverter, a control board with a TMS320F28335 DSP, an incremental encoder, two Hall-effect current sensors, and a load motor. The dc-link voltage (295 VDC) is obtained from the utility (AC 220V/60Hz) using a single-phase full-bridge rectifier. The two phase currents $\left(i_{a}, i_{b}\right)$ are measured by LTS6-NP Hall Sensors and then converted into digital form using two 12-bit A/D converters. In addition, the rotor position $(\theta)$, which is used to execute the coordinate transformation in the field-oriented control (FOC) technique, is measured by a RIA-40-2500ZO encoder and fed to Texas Instruments TMS320F28335 DSP via a 32-bit QEP. Note that the measured rotor speed $(\omega)$ required to perform the feedback control can be easily obtained by differentiating $\theta$ with respect to time. Moreover, the control inputs $V_{q s}$ and $V_{d s}$, 


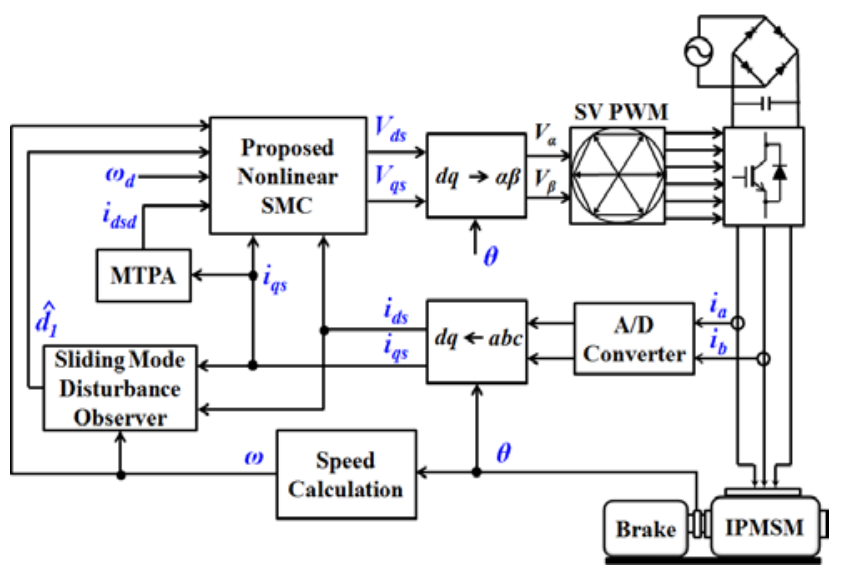

Fig. 1. Overall block diagram of the proposed observer-based nonlinear sliding mode speed control system.

which are given by the proposed observer-based control algorithm, are transformed to $V_{\alpha}$ and $V_{\beta}$ in the stationary $\alpha-\beta$ reference frame. Then, the space vector PWM (SVPWM) block generates six gating signals that drive a three-phase IGBT smart power module FSBS5CH60 (600V/5A). In this paper, the sampling frequency $\left(T_{s}\right)$ and the PWM switching frequency $\left(f_{s}\right)$ are chosen as $200 \mu$ s and $5 \mathrm{kHz}$, respectively, with the system efficiency and control performance taken into account.

In this study, the following two cases are implemented to validate the effectiveness of the proposed observer-based nonlinear SMC scheme:

- Case 1: The desired rotor speed $\left(\omega_{d}\right)$ abruptly accelerates from $1000 \mathrm{rpm}$ to $1500 \mathrm{rpm}$ while the load torque $\left(T_{L}\right)$ is kept constant at $1 \mathrm{~N} \cdot \mathrm{m}$, with electrical motor parameter variations $\left(\Delta R_{s}=\right.$ $\left.+0.5 R_{s}, \Delta L_{d s}=+0.1 L_{d s}, \Delta L_{q s}=-0.3 L_{q s}\right)$.

- Case 2: The load torque $\left(T_{L}\right)$ suddenly changes from $0.5 \mathrm{~N} \cdot \mathrm{m}$ to $1.0 \mathrm{~N} \cdot \mathrm{m}$ while the desired speed $\left(\omega_{d}\right)$ is kept constant at $100 \mathrm{rpm}$, with mechanical motor parameter variations $(\Delta J=+2 J, \Delta B=$ $+1.5 B)$.

It should be pointed out that Cases 1 and 2 are given to demonstrate the robustness of the proposed observer-based nonlinear SMC strategy. Actually, it is not easy to straightforwardly implement Cases 1 and 2 in real experiments. Thus, changing the parameters of the control scheme can be an alternative to directly modify the motor parameters in actual motor drives. In this paper, the motor parameters of the controller are indirectly changed rather than those of the real motor drives in order to perform the experiments on the proposed observer-based nonlinear SMC strategy under variations in the electrical parameters and the mechanical parameters.

Figs. 2 and 3 show the experimental results of the proposed observer-based nonlinear SMC method under Cases 1 and 2, respectively. Each plot shows the following: (a) The desired

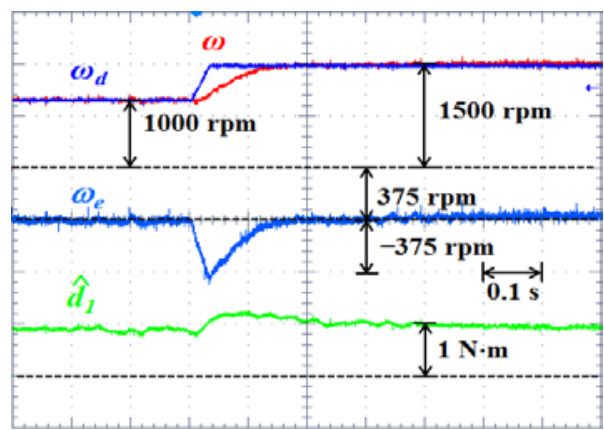

(a)

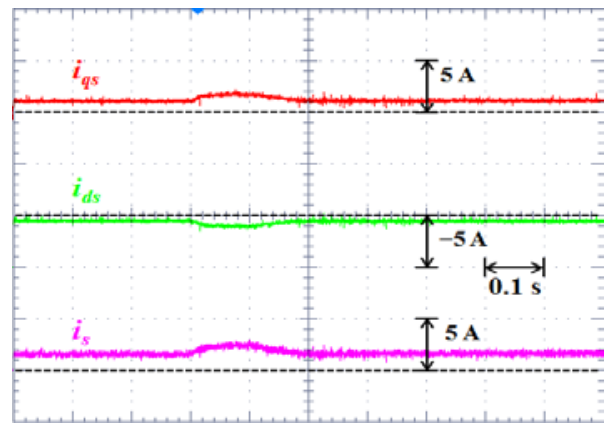

(b)

Fig. 2. Experimental results of the proposed observer-based nonlinear SMC under Case 1. (a) $\omega_{d}, \omega, \omega_{e}$, and $\hat{d}_{1}$. (b) $i_{q s}, i_{d s}$, and $i_{s}$.

TABLE I

Performance Comparison of Two Control Methods BASED ON EXPERIMENTAL RESULTS

\begin{tabular}{|c|c|c|c|c|}
\hline \multirow{2}{*}{ Items } & \multicolumn{2}{|c|}{ Settling time [ms] } & \multicolumn{2}{c|}{ Speed error [rpm] } \\
\cline { 2 - 5 } & $\begin{array}{c}\text { Nonlinear } \\
\text { SMC }\end{array}$ & $\begin{array}{c}\text { Linear } \\
\text { SMC }\end{array}$ & $\begin{array}{c}\text { Nonlinear } \\
\text { SMC }\end{array}$ & $\begin{array}{c}\text { Linear } \\
\text { SMC }\end{array}$ \\
\hline 1 & 90 & 130 & 17 & 21 \\
\hline 2 & 30 & 60 & 4 & 13 \\
\hline
\end{tabular}

rotor speed $\left(\omega_{d}\right)$, actual rotor speed $(\omega)$, speed error $\left(\omega_{e}\right)$, and estimated system uncertainties $\left(\hat{d}_{1}\right)$; (b) The actual $q$-axis current $\left(i_{q s}\right)$, actual $d$-axis current $\left(i_{d s}\right)$, and stator current amplitude $\left(i_{s}\right)$. Fig. 2 depicts the dynamic responses of the rotor speed (Cases 1). In this case, the control system achieves good speed performance with a fast response (a settling time of about $90 \mathrm{~ms}$ ) and almost zero steady-state errors under a sudden change in speed from $1000 \mathrm{rpm}$ to $1500 \mathrm{rpm}$. Fig. 3 shows the rotor speed stability (Case 2) when the load torque varies with a step change. In this figure, it can be observed that the proposed control scheme gives a rapid and precise estimation of the uncertainties and maintains a stable speed response during a sudden change in the load torque (i.e. from $0.5 \mathrm{~N} \cdot \mathrm{m}$ to $1.0 \mathrm{~N} \cdot \mathrm{m}$ ).

To compare the performances of the proposed observer-based nonlinear sliding mode controller, a corresponding observer-based linear sliding mode controller is implemented as follows:

$$
\sigma_{l}=\left[\begin{array}{ll}
F & I
\end{array}\right] \hat{z}=S_{l} \hat{z}
$$




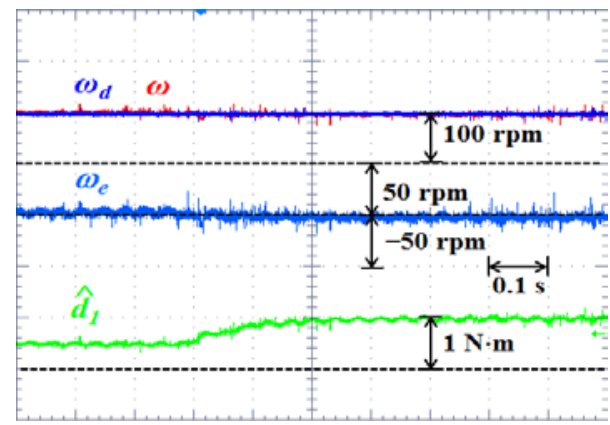

(a)

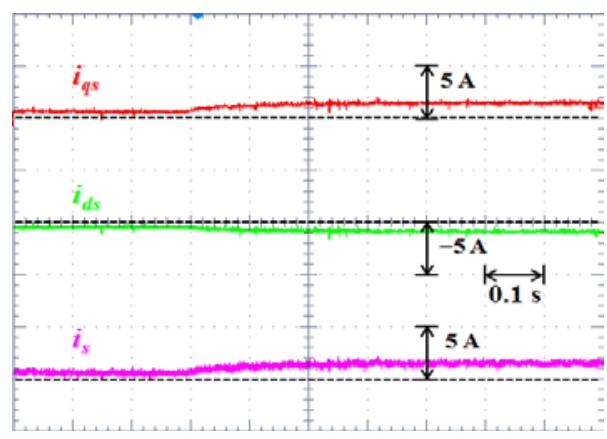

(b)

Fig. 3. Experimental results of the proposed observer-based nonlinear SMC under Case 2. (a) $\omega_{d}, \omega, \omega_{e}$, and $\hat{d}_{1}$. (b) $i_{q s}, i_{d s}$, and $i_{s}$.

$$
\hat{u}_{l}=\left[\begin{array}{l}
\hat{u}_{l 1} \\
\hat{u}_{l 2}
\end{array}\right]=-\left(S_{l} A \hat{z}+K \hat{\sigma}_{l}+\hat{\gamma} \frac{\hat{\sigma}_{l}}{\left\|\hat{\sigma}_{l}\right\|+\eta}\right)
$$

Consequently, the control input signals applied to the space vector pulse-width modulation (SVPWM) are given by:

$$
V_{q s}=\frac{1}{k_{6}} \hat{u}_{1 l}, \quad V_{d s}=\frac{1}{k_{8}} \hat{u}_{2 l}
$$

Figs. 4 and 5 show the experimental results of the corresponding observer-based linear SMC method under the same conditions (Cases 1 and 2) as the proposed observer-based nonlinear SMC method. Since the sliding surface gain (F) given in (51) is obtained with a fixed initial settling time and damping ratio, compared with Fig. 2, the speed responses in Fig. 4 are much slower (about $130 \mathrm{~ms}$ ) and indicate bigger steady-state errors (21 rpm). In Fig. 5, the steady-state speed error (13 rpm) is much bigger than the one (4 rpm) in Fig. 3 when the load torque is suddenly applied, i.e., from $0.5 \mathrm{~N} \cdot \mathrm{m}$ to $1.0 \mathrm{~N} \cdot \mathrm{m}$. Table I summarizes the performance comparison between the proposed observer-based nonlinear SMC and the corresponding observer-based linear SMC based on the experimental results of Figs. 2-5.

Note that Figs. 2 to 5 indicate the experimental results of the proposed and conventional control methods with the MTPA operation as shown in (9). In order to demonstrate the effectiveness of the MTPA control, the proposed control

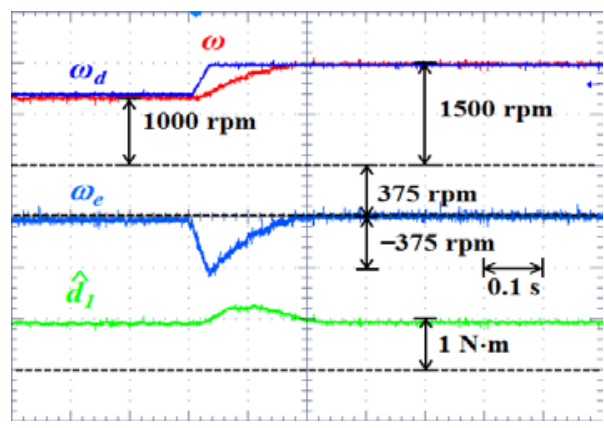

(a)

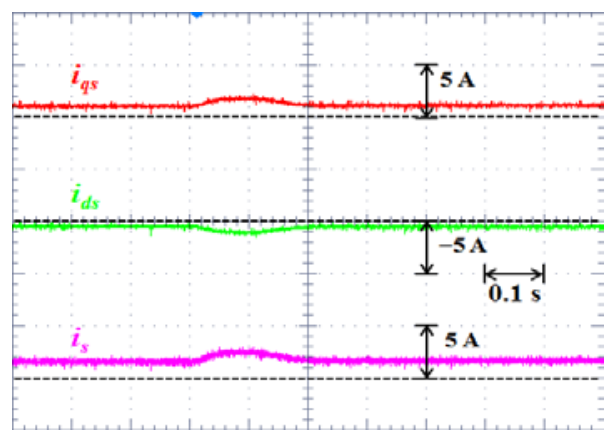

(b)

Fig. 4. Experimental results of the corresponding observer-based linear SMC under Case 1. (a) $\omega_{d}, \omega, \omega_{e}$, and $\hat{d}_{1}$. (b) $i_{q s}, i_{d s}$, and $i_{s}$.

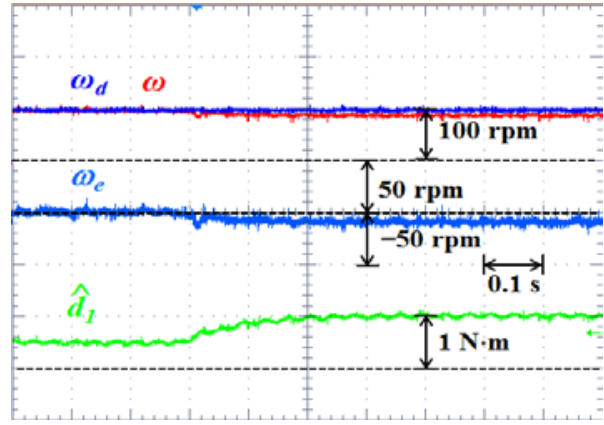

(a)

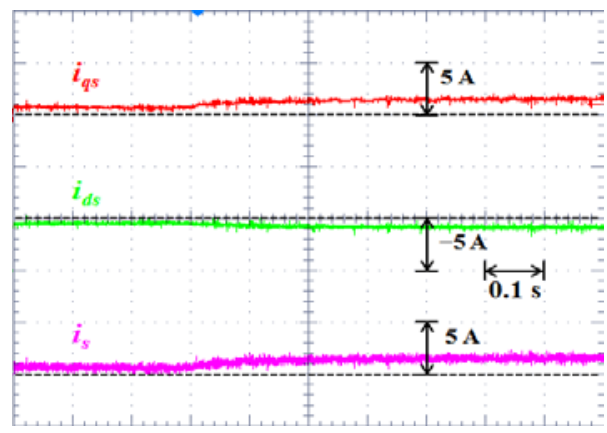

(b)

Fig. 5. Experimental results of the corresponding observer-based linear SMC under Case 2. (a) $\omega_{d}, \omega, \omega_{e}$, and $\hat{d}_{1}$. (b) $i_{q s}, i_{d s}$, and $i_{s}$.

method without the MTPA operation is carried out under Case 1 . In this case, the desired $d$-axis current is set to zero. It 


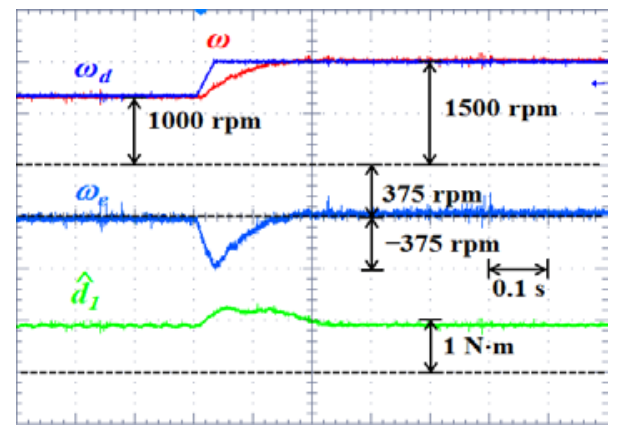

(a)

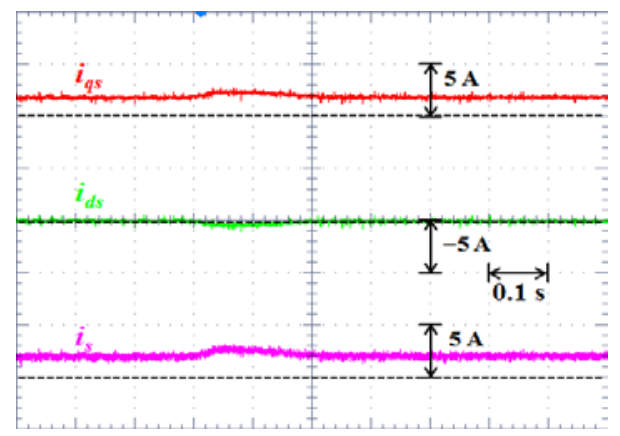

(b)

Fig. 6. Experimental results of the proposed observer-based nonlinear SMC without MTPA control under Case 1. (a) $\omega_{d}, \omega$, $\omega_{e}$, and $\hat{d}_{1}$. (b) $i_{q s}, i_{d s}$, and $i_{s}$.

can be seen that the transient responses and the steady-state speed errors of the proposed control scheme with the MTPA operation (Fig. 2) and without the MTPA operation (Fig. 6) are almost the same. However, due to the ability of the MTPA control technique to minimize the stator current to generate the maximum torque, the stator current amplitude $\left(i_{s}=1.8 \mathrm{~A}\right)$ in Fig. 2 is smaller than that $\left(i_{s}=2.0 \mathrm{~A}\right)$ in Fig. 6. This leads to optimal system efficiency by minimizing losses such as the copper losses of the stator windings and the conduction losses of the power switches. It is noted that the experimental results of the proposed control method without the MTPA operation are given under only Case 1 due to space limitations.

From the presented experimental results it can be observed that the speed responses of the proposed nonlinear SMC are faster than those of the corresponding linear SMC under system parameter variations. In addition, the speed tracking ability of the proposed nonlinear SMC is significantly better than that of the corresponding linear SMC with small steady-state errors in the presence of parameter uncertainties.

\section{CONCLUSION}

This paper proposed a robust sliding mode speed controller for SPMSM drives to achieve fast and precise speed tracking. By combining a FNNC term and an adaptive SMC term, the proposed controller did not require any knowledge of the nominal system parameters. Therefore, it was very robust to system parameter variations. Based on the Lyapunov theory and the related lemmas, a stability evaluation of the proposed SMC system was presented in detail. For a comparison, both the proposed SMC control scheme and a conventional SMC scheme were simulated and tested under motor parameter variations. Through these experimental results, it was verified that the proposed SMC method achieved superior control performance (i.e., faster and more robust dynamic behavior and smaller steady-state errors) when compared to the conventional SMC method.

\section{ACKNOWLEDGMENT}

This work was supported by the research program of Dongguk University, 2014.

\section{REFERENCES}

[1] H. W. Sim, J. S. Lee, and K. B. Lee, “On-line parameter estimation of interior permanent magnet synchronous motor using an extended Kalman filter," J. Electr. Eng. Technol., Vol. 9, No. 2, pp. 600-608, Mar. 2014.

[2] M. Sekour, K. Hartani, A. Draou, and A. Allali, "Sensorless fuzzy direct torque control for high performance electric vehicle with four in-wheel motors," $J$. Electr. Eng. Technol., Vol. 8, No. 3, pp. 530-543, May 2013.

[3] H. Lin, K. Y. Hwang, and B. I. Kwon, “An improved flux observer for sensorless permanent magnet synchronous motor drives with parameter identification,” J. Electr. Eng. Technol., Vol. 8, No. 3, pp. 516-523, May 2013.

[4] T. D. Do, S. Kwak, H. H. Choi, and J. W. Jung, "Suboptimal control scheme design for interior permanentmagnet synchronous motors: an SDRE-based approach," IEEE Trans. Power Electron., Vol. 29, No. 6, pp. 3020-3031, Jun. 2014.

[5] D. Q. Dang, N. T. T. Vu, H. H. Choi, and J. W. Jung, "Neural-fuzzy control of interior permanent magnet synchronous motor: stability analysis and implementation," J. Electr. Eng. Technol., Vol. 8, No. 6, pp. 1439-1450, Nov. 2013.

[6] S. Li and H. Gu, "Fuzzy adaptive internal model control schemes for PMSM speed-regulation system,” IEEE Trans. Ind. Informat., Vol. 8, No. 4, pp. 767-779, Nov. 2012.

[7] Y. A. R. I. Mohamed and T. K. Lee, "Adaptive self-tuning MTPA vector controller for IPMSM drive system," IEEE Trans. Energy Convers., Vol. 21, No. 3, pp. 636-644, Sep. 2006.

[8] M. N. Uddin and R. S. Rebeiro, "Online efficiency optimization of a fuzzy-logic-controller-based IPMSM drive," IEEE Trans. Ind. Appl., Vol. 47, No. 2, pp. 1043-1050 , Mar./Apr. 2011.

[9] M. N. Uddin and M. A. Rahman, "High-speed control of IPMSM drives using improved fuzzy logic algorithms," IEEE Trans. Ind. Electron., Vol. 54, No. 1, pp. 190-199, Feb. 2007.

[10] M. A. S. Khan and M. A. Rahman, "A novel neuro-wavelet-based self-tuned wavelet controller for IPM motor drives,” IEEE Trans. Ind. Appl., Vol. 46, No. 3, pp. 1194-1203, May/Jun. 2010.

[11] J. Yang, S. Li, J. Su, and X. Yu, “Continuous nonsingular terminal sliding mode control for systems with mismatched 
disturbances,” Automatica, Vol. 49, No. 7, pp. 2287-2291, Apr. 2013.

[12] C. E. Castaneda, A. G. Loukianov, E. N. Sanchez, and B. Castiio-Toledo, "Discrete-time neural sliding-mode block control for a DC motor with controlled flux," IEEE Trans. Ind. Electron., Vol. 59, No. 2, pp. 1194-1207, Feb. 2012.

[13] B. Veselic, B. Perunicic-Drazenovic, and C. Milosavljevic, "High-performance position control of induction motor using discrete-time sliding-mode control," IEEE Trans. Ind. Electron., Vol. 55, No. 11, pp. 3809-3817, Nov. 2008.

[14] F. Lin, Y. Hung, and M. Tsai, "Fault tolerant control for six-phase PMSM drive system via intelligent complementary sliding mode control using TSKFNN-AMF,” IEEE Trans. Ind. Electron., Vol. 60, No. 12, pp. 5747-5762, Dec. 2013.

[15] V. Q. Leu, H. H. Choi, and J. W. Jung, "Fuzzy sliding mode speed controller for PM synchronous motors with a load torque observer," IEEE Trans. Power Electron., Vol. 27, No. 3, pp. 1530-1539, Mar. 2012.

[16] J. W. Jung, V. Q. Leu, D. Q. Dang, H. H. Choi, and T. H. Kim, "Sliding mode control of SPMSM drivers: an online gain tuning approach with unknown system parameters," Journal of Power Electronics, Vol. 14, No. 5, pp. 980-988, Sep. 2014.

[17] D. Fulwani, B. Bandyopadhyay, and L. Fridman, "Non-linear sliding surface: towards high performance robust control," IET Control Theory Appl., Vol. 6, No. 2, pp. 235-242, Jan. 2012.

[18] B. M. Chen, T. H. Lee, K. Peng, and V. Venkataramanan, "Composite nonlinear feedback control for linear systems with input saturation: theory and applications,” IEEE Trans. Autom. Control, Vol. 48, No. 3, pp. 427-439, Mar. 2003.

[19] V. I. Utkin, Sliding Modes in Control Optimization, Springer-Verlag, 1992.

[20] J. Yang, J. Su, S. Li, and X. Yu, "High-order mismatched disturbance compensation for motion control systems via a continuous dynamic sliding-mode approach," IEEE Trans. Ind. Inform., Vol. 10, No. 1, pp. 604-614, Feb. 2014.

[21] J. Yang, S. Li, and X. Yu, "Sliding-mode control for systems with mismatched uncertainties via a disturbance observer. IEEE Trans. Ind. Electron., Vol. 60, No. 1, pp. 160-169, Jan. 2013.

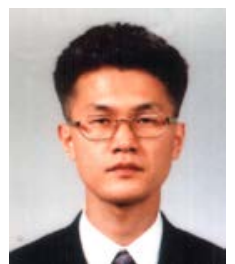

Jin-Woo Jung received his B.S. and M.S. degrees in Electrical Engineering from Hanyang University, Seoul, Korea, in 1991 and 1997, respectively. He received his Ph.D. degree in Electrical and Computer Engineering from the Ohio State University, Columbus, Ohio, USA, in 2005. From 1997 to 2000, he was with the Home Appliance Research Laboratory, LG Electronics Co., Ltd., Seoul, Korea. From 2005 to 2008, he worked as a Senior Engineer at the R\&D Center and with the PDP Development Team, Samsung SDI Co., Ltd., Korea. Since 2008, he has been an Associate Professor in the Division of Electronics and Electrical Engineering, Dongguk University, Seoul, Korea. His current research interests include DSP-based electric machine drives, distributed generation systems using renewable energy sources, and power conversion systems and drives for electric vehicles (EVs).

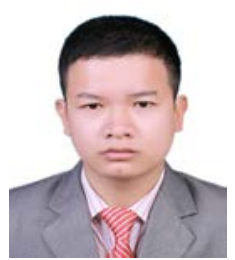

Dong Quang Dang received his B.S. and M.S. degrees in Electrical Engineering from the Hanoi University of Science and Technology (HUST), Hanoi, Vietnam, in 2005 and 2010, respectively. He received his Ph.D. degree from the Division of Electronics and Electrical Engineering,
Dongguk University, Seoul, Korea, 2015. From 2006 to 2011, he worked as a Lecturer at the Hung Yen University of Technology and Education, Hung Yen, Vietnam. His current research interests include electric machine drives and distributed generation systems using renewable energy sources.

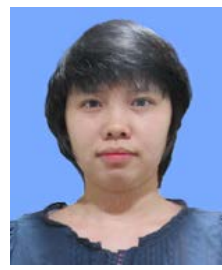

Nga Thi-Thuy Vu received her B.S. and M.S. degrees in Electrical Engineering from the Hanoi University of Science and Technology (HUST), Hanoi, Vietnam, in 2005 and 2008, respectively. She received her Ph.D. degree from the Division of Electronics and Electrical Engineering, Dongguk University, Seoul, Korea, 2013. From 2008 to 2009, she was a Lecturer at the Water Resources University, Hanoi, Vietnam. Her current research interests include DSP-based electric machine drives and distributed generation systems using renewable energy sources.

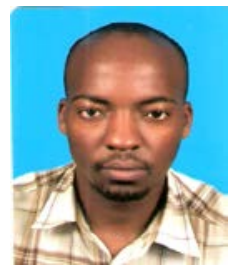

Jackson John Justo received his B.S. degree in Electrical Engineering from the University of Dar es Salaam, Dar es Salaam, Tanzania, in 2008. He received his Ph.D. degree from the Division of Electronics and Electrical Engineering, Dongguk University, Seoul, Korea, 2015. He also worked as a member of the academic staff in the Department of Electrical Engineering, University of Dar es Salaam. His current research interests include distributed generation systems, nano-grid and micro-grid systems, electric vehicles (EVs), and electric machine drives.

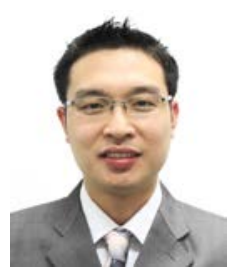

Ton Duc Do received his B.S. and M.S. degrees in Electrical Engineering from the Hanoi University of Science and Technology (HUST), Hanoi, Vietnam, in 2007 and 2009, respectively. He received his Ph.D. degree from the Division of Electronics and Electrical Engineering, Dongguk University, Seoul, Korea, in 2014. From 2008 to 2009, he worked as a Lecturer at the Water Resources University, Hanoi, Vietnam. His current research interests include electric machine drives and distributed generation systems using renewable energy sources.

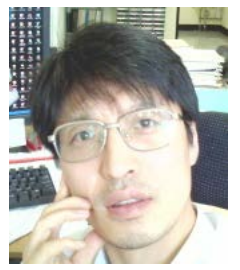

Han Ho Choi received his B.S. degree in Control and Instrumentation Engineering from Seoul National University (SNU), Seoul, Korea, in 1988, and his M.S. and Ph.D. degrees in Electrical Engineering from the Korea Advanced Institute of Science and Technology (KAIST), Daejeon, Korea, in 1990 and 1994, respectively. He is presently with the Division of Electronics and Electrical Engineering, Dongguk University, Seoul, Korea. His current research interests include control theory and its application to real world problems.

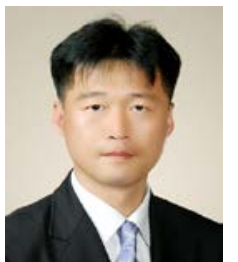

Tae Heoung Kim received his B.S., M.S., and Ph.D. degrees in Electrical Engineering from Hanyang University, Seoul, Korea, in 1993, 1995, and 2005, respectively. From 1995 to 2002, he worked as a Senior Research Engineer for LG Electronics, Korea. Since 2005, he has been a Professor in the Department of Electrical Engineering, Gyeongsang National University, Jinju, Korea. His current research interests include the design of electric machines and control systems. 